\title{
3. \\ Beweis einiger geometrischen Sätze.
}

(Von Herrn Th. Clausen zu München.)

1. Es seien die Coordinaten dreier Puncte $M_{0}, M_{1}, M_{2}$, in einer Ebene $x, y ; x^{\prime}, y^{\prime} ; x^{\prime \prime}, y^{\prime \prime} ;$ bestimmt man nun 6 andere Puncte $A_{0}, A_{1}, A_{2}$ und $J_{0}, J_{1}, J_{2}$, deren Coordinaten resp. $\xi_{0}, \xi_{1}, \xi_{2},{ }_{0} \xi,{ }_{2} \xi$, ${ }_{2} \xi$, und $v_{0}, v_{1}$, $v_{2},{ }_{0}^{v},{ }_{2} v,{ }_{2} v$, sind, wo

$$
\begin{array}{ll}
\xi_{0}=\frac{\alpha^{\prime} x^{\prime}-\alpha^{\prime \prime} x^{\prime \prime}}{\alpha^{\prime}-\alpha^{\prime \prime} ;} & v_{0}=\frac{\alpha^{\prime} y^{\prime}-\alpha^{\prime \prime} y^{\prime \prime}}{\alpha^{\prime}-\alpha^{\prime \prime}} ; \\
\xi_{1}=\frac{\alpha^{\prime \prime} x^{\prime \prime}-\alpha x}{\alpha^{\prime \prime}-\alpha} ; & v_{1}=\frac{\alpha^{\prime \prime} y^{\prime \prime}-\alpha y}{\alpha^{\prime \prime}-\alpha} ; \\
\xi_{2}=\frac{\alpha x-\alpha^{\prime} x^{\prime}}{\alpha-\alpha^{\prime}} ; & v_{2}=\frac{\alpha y-\alpha^{\prime} y^{\prime}}{\alpha-\alpha^{\prime}} ; \\
{ }_{0} \xi=\frac{\alpha^{\prime} x^{\prime}+\alpha^{\prime \prime} x^{\prime \prime}}{\alpha^{\prime}+\alpha^{\prime \prime}} ; & { }_{0} v=\frac{\alpha^{\prime} y^{\prime}+\alpha^{\prime \prime} y^{\prime \prime}}{\alpha^{\prime}+\alpha^{\prime \prime}} ; \\
{ }_{1} \xi=\frac{\alpha^{\prime \prime} x^{\prime \prime}+\alpha^{\prime} x}{\alpha^{\prime \prime}+\alpha} ; & { }_{{ }^{v}}=\frac{\alpha^{\prime \prime} y^{\prime \prime}+\alpha y}{\alpha^{\prime \prime}+\alpha} ; \\
{ }_{2} \xi=\frac{\alpha x+\alpha^{\prime} x^{\prime}}{\alpha+\alpha^{\prime}} ; & { }_{2} v=\frac{\alpha y+\alpha^{\prime} y^{\prime}}{\alpha+\alpha^{\prime}} ;
\end{array}
$$

so finden zwischen diesen 9 Puncten folgende Relationen Statt.

2. Je drei derselben liegen folgendermafsen in einer Geraden:

$$
\begin{array}{ccc}
A_{0}, & A_{1}, & A_{2} ; \\
A_{0}, & J_{1}, & J_{2} ; \\
J_{0}, & A_{1}, & J_{2} ; \\
J_{0}, & J_{1}, & A_{2} ;
\end{array}
$$

denn es seien die Coordinaten dreier in einer Geraden liegenden Puncte $\xi, \xi^{\prime}, \xi^{\prime \prime}$ und $v, u^{\prime}, v^{\prime \prime}$, und die allgemeine Gleichung für dieselbe

so hat man:

$$
a x+b y+c=0
$$

$$
\begin{aligned}
& a \xi+b v+c=0, \\
& a \xi^{\prime}+b v^{\prime}+c=0, \\
& a \xi^{\prime \prime}+b v^{\prime \prime}+c=0 .
\end{aligned}
$$

Eliminirt man hieraus die die Lage der Linie bestimmenden Constanten, indem man sie resp. mit den Factoren $m, m^{\prime}, m^{\prime \prime}$ multiplicirt und die Producte addirt, so findet man folgende Bedingungen für die Coordinaten 
dreier in einer Geraden liegenden Puncte:

$$
\begin{aligned}
& m \xi+m^{\prime} \xi^{\prime}+m^{\prime \prime} \xi^{\prime \prime}=0, \\
& m v+m^{\prime} v^{\prime}+m^{\prime \prime} v^{\prime \prime}=0, \\
& m+m^{\prime}+m^{\prime \prime}=0,
\end{aligned}
$$

Diese Bedingungen sind offenbar hinsiehtlich der drei Puncte $A_{0}, A_{1}, A_{2}$ erfüllt, wenn man $m, m^{\prime}, m^{\prime \prime}$ resp. $\alpha^{\prime}-\alpha^{\prime \prime}, \alpha^{\prime \prime}-\alpha, \alpha-\alpha^{\prime \prime}$ setzt; und eben so für die drei übrigen mal drei Puncte, indem blos $-\alpha$ statt $+\alpha$ für die ersten drei folgenden, denn $-\alpha^{\prime}$ statt $+\alpha^{\prime}$, und $-\alpha^{\prime \prime}$ statt $+\alpha^{\prime \prime}$ für die letzten gesetzt wird.

3. Nachstehende zu drei und drei nebeneinanderstehende Linien sehneiden sich in einem Puncte:

$$
\begin{array}{lll}
M_{\mathrm{o}} J_{\mathrm{o}}, & M_{\mathrm{1}} J_{1}, & M_{2} J_{2} ; \\
M_{\mathrm{o}} J_{\mathrm{o}}, & M_{1} A_{1}, & M_{2} A_{2} ; \\
M_{\mathrm{o}} A_{\mathrm{o}}, & M_{1} J_{1}, & M_{2} A_{2} \\
M_{\mathrm{o}} A_{\mathrm{o}}, & M_{1} A_{1}, & M_{\mathrm{2}} J_{2} ;
\end{array}
$$

denn es seien von irgend einer Linie zwei Puncte gegeben, deren Coordinaten resp. $\xi$, $\xi^{\prime}$ und $v, v^{\prime}$ sind, so kann man die Coordinaten jedes Punctes derselben durch

$$
p \xi+(1-p) \xi^{\prime} ; p v+(1-p) v^{\prime}
$$

darstellen, wo $p$ veränderlich ist. Die Coordinaten der Linien $M_{\mathrm{o}} J_{0}$, $M_{1} J_{\mathrm{y}}, M_{2} J_{2}$ können also so dargestellt werden :

$$
\begin{array}{ll}
p_{0} x+\left(1-p_{0}\right)_{0} \xi ; & p_{0} y+\left(1-p_{0}\right)_{0} v ; \\
p_{2} x^{\prime}+\left(1-p_{2}\right)_{1} \xi ; & p_{1} y^{\prime}+\left(1-p_{1}\right)_{2} v ; \\
p_{2} x^{\prime \prime}+\left(1-p_{2}\right)_{2} \xi ; & p_{2} y^{\prime \prime}+\left(1-p_{2}\right)_{2} v ;
\end{array}
$$

giebt es nun für $p_{0}, p_{1}, p_{2}$ solche Werthe, dafs die resp. Coordinaten für alle drei Puncte gleich sind, so schneiden sie sich offenbar in diesem Puncte. Setzt man aber:

$$
p_{0}=\frac{\alpha}{\alpha+\alpha^{\prime}+a^{\prime \prime}} ; \quad p_{1}=\frac{\alpha^{\prime}}{\alpha+\alpha^{\prime}+\alpha^{\prime \prime}} ; \quad p_{2}=\frac{\alpha^{\prime \prime}}{\alpha+\alpha^{\prime}+\alpha^{\prime \prime}} ;
$$

und substituirt in obige Coordinaten statt ${ }_{0} \xi,{ }_{1} \xi,{ }_{2} \xi$ ihre Werthe, so folgen für alle drei Puncte die gleichen Cooriinaten:

$$
\frac{\alpha x+\alpha^{\prime} x^{\prime}+\alpha^{\prime \prime} x^{\prime \prime}}{\alpha+\alpha^{\prime}+\alpha^{\prime \prime}} \text { und } \frac{\alpha y+\alpha^{\prime} y^{\prime}+\alpha^{\prime \prime} y^{\prime \prime}}{a+\alpha^{\prime}+c^{\prime \prime}},
$$

was zu beweisen war. Setzt man nun statt $\alpha$, - , so ergiebt sich der Beweis, dafs die Linien $M_{0} J_{0}, M_{1} A_{1}, M_{8} A_{2}$ sieh ebenfalls in einem Puncte schneiden, uud durch Verwechselung von $+\alpha^{\prime}$ und $-\alpha^{\prime}$, und $+\alpha^{\prime \prime}$ und - $a^{\prime \prime}$ für die zwei übrigen mal drei Linien. 
4. Nimmt man an, dafs auf die drei Puncte der wagerechten Ebene $M_{0}, M_{1}, M_{2}$ die resp. Kräfte $+\alpha$ und $-\alpha ;+\alpha^{\prime}$ und $-\alpha^{\prime} ;+\alpha^{\prime \prime}$ und - $a^{\prime \prime}$ senkrecht wirken, so ist die Ebene offenbar im Gleichgewicht. Sieht man nun jede verlingerte Seite des Dreiecks $M_{0}, M_{1}, M_{2}$ als einen Hebel an, auf den in jedem der zwei sie bestimmenden Puncte eine der in denselben angebrachten Kräfte wirkt, dann sind die 6 oben bestimmten Puncte offenbar alle Stützpunote dieser verschiedenen Hebel. Diese Betrachtung giebt ein Mittel die obigen Sätze aus den Grundsätzen der Statik abzuleiten.

Verbindet man nemlich $+\alpha^{\prime}$ und $-\alpha^{\prime \prime} ;+\alpha^{\prime \prime}$ und $-\alpha ;+\alpha$ und - $a^{\prime}$, so sind die drei Stützpuncte auf die man die resp. Summen der Kräfte als wirkend ansehen kann $A_{\mathrm{o}}, A_{1}, A_{\mathrm{g}}$. Da aber die Ebene durch diese 6 Kräfte im Gleichgewicht ist, so mufs jede dieser drei Puncte zu den zwei übrigen der Stützpunct sein, und also mit ihnen in gerader Linie liegen. Man sieht leicht, wie man die Kräfte vertheilen mufs, um den Beweis für die drei übrigen mal drei Puncte zu führen.

5. Es wirken auf die drei Puncte die resp. Kräfte $+a,+\alpha^{\prime}$, $+\alpha^{\prime \prime}$. Man kann nun die beiden Kräfte $\alpha^{\prime}$ und $\alpha^{\prime \prime}$ als in ihrem Stätzpuncte $J_{0}$ angebracht ansehen. Der Stützpunct für alle drei Kräfte liegt also in der Linie $M_{0} J_{0}$. Eben so findet man, dafs er in den Linien $M_{1} J_{1}$ und $M_{2} J_{2}$ liege. Diese drei Linien müssen sich also in einem Puncte schneiden. Ist in dem Punct $M_{\mathrm{o}}$ statt $+\alpha$ die Kraft - $\alpha$ angebracht, so findet man, dafs der Stützpunct der drei Kräfte auf den Linien $M_{\mathrm{o}} J_{\mathrm{o}}$, $M_{2} A_{1}, M_{2} A_{2}$ liege, die sich also auch in einem Puncte schneiden. Eben so ergiebt sich der Beweis für die beiden übrigen mal drei Linien.

6. Beschreibt man aus den Puncten $M_{0}, M_{1}, M_{2}$ mit den resp. Halbmessern $\frac{k}{\alpha}, \frac{k}{\alpha^{\prime}}, \frac{k}{\alpha^{\prime \prime}}$ drei Kreise, wo $k$ eine willkürliche Gröfse bedeutet, so sind die benannten 6 Puncte die Durchschnitte der durch die Endpuncte paralleler Radien je zweier dieser Kreise gezogenen Geraden; oder nach Hrn. St einer's Benennung (Gegenw. Journal Bd. I. p. 172.) die Ähnlichkeitspuncte derselben. Diese haben also die in 2. und 3. bewiesenen Relationen, von denen Hr. Ste in er jene aus seinen sinnreichen geometrischen Betrachtungen abgeleitet hat.

7. Setzt man $\alpha=\alpha^{\prime}=\alpha^{\prime \prime}$, so sind die Puncte $J_{o}, J_{1}, J_{2}$ die Mitten der Seiten des Dreiecks. Zieht man also von diesen Puncten aus 
nach den gegenüberstehenden Spitzen Geraden, so schneiden sich diese nach 3 in einem Puncte.

8. Sind die Winkel des Dreiecks $M_{0} M_{1} M_{2}, \varphi, \varphi^{\prime}, \varphi^{\prime \prime}$ und setzt man : $\alpha=\sin \varphi, \quad \alpha^{\prime}=\sin \varphi^{\prime}, \quad \alpha^{\prime \prime}=\sin \varphi^{\prime \prime}$,

so sind die 6 Puncte, wie leicht zu sehen ist, die Durchschnitte der die innern und äufsern Winkel des Dreiecks halbirenden Geraden mit den gegenüberstehenden Seiten. Für diese gelten also auch die in 2. und 3. bewiesenen Relationen. (S. Gegenw. Journal Bd. II. p. 191. Lehrsatz 33. No. 2.)

9. Setzt man:

$$
\alpha=\operatorname{tang} \varphi, \quad \alpha^{\prime}=\operatorname{tang} \varphi^{\prime}, \quad \alpha^{\prime \prime}=\operatorname{tang} \varphi^{\prime \prime},
$$

dann sind $J_{0}, J_{1}, J_{2}$ die Fufspuncte der auf die Seiten des Dreiecks aus den gegenüberstehenden Spitzen gefällten Perpendikel. Diese schneiden sich also nach 3 . in einem Puncte.

10. Es ist:

$$
\begin{aligned}
& \alpha M_{\mathrm{o}} J_{2}=\alpha^{\prime} M_{1} J_{2} ; \\
& \alpha^{\prime} M_{1} J_{\mathrm{o}}=\alpha^{\prime \prime} M_{\mathrm{q}} J_{\mathrm{o}} ; \\
& \alpha^{\prime \prime} M_{\mathrm{2}} J_{1}=\alpha M_{\mathrm{o}} J_{1} ;
\end{aligned}
$$

folglich :

$$
M_{\mathrm{o}} J_{2} \cdot M_{1} J_{\mathrm{o}} \cdot M_{\mathrm{2}} J_{1}=M_{1} J_{2} \cdot M_{2} J_{\mathrm{o}} \cdot M_{\mathrm{o}} J_{1} \text {, }
$$

welchen Satz ich irgendwo gesehen zu haben mich erinnere.

11. Der Inhalt der Dreiecke $J_{0} M_{1} J_{2}, J_{1} M_{2} J_{v}, J_{2} M_{0} J_{1}, J_{0} J_{2} J_{2}$ sei resp. $i_{1}, i_{2}, i_{0}, g$ und das Dreieck $M_{0} M_{1} M_{2}=\triangle$, so ist, wie leicht zu übersehen,

$$
\begin{aligned}
i_{1}=\frac{\alpha^{\prime \prime} \alpha}{\left(\alpha+\alpha^{\prime}\right)\left(\alpha^{\prime}+\alpha^{\prime \prime}\right)} \cdot \Delta ; & i_{2}=\frac{\alpha \alpha^{\prime}}{\left(\alpha^{\prime}+\alpha^{\prime \prime}\right)\left(\alpha^{\prime \prime}+\alpha\right)} \cdot \Delta ; i=\frac{\alpha^{\prime} \alpha^{\prime \prime}}{\left(\alpha^{\prime \prime}+\alpha\right)\left(\alpha+\alpha^{\prime}\right)} \cdot \Delta ; \\
& i_{0}+i_{2}+i_{2}+g=\Delta .
\end{aligned}
$$

Es wird demnach:

$\left(\alpha+\alpha^{\prime}\right)\left(\alpha^{\prime}+\alpha^{\prime \prime}\right)\left(\alpha^{\prime \prime}+\alpha\right)\left(i_{0}+i_{1}+i_{2}\right)=\left(\alpha \alpha^{\prime 2}+\alpha^{\prime} \alpha^{\prime \prime 2}+\alpha^{\prime \prime} \alpha^{2}+\alpha^{2} \alpha^{\prime}+\alpha^{\prime 2} \alpha^{\prime \prime}+\alpha^{\prime \prime 2} \alpha^{\prime}\right) \Delta$, $\left(\alpha+\alpha^{\prime}\right)\left(\alpha^{\prime}+a^{\prime \prime}\right)\left(\alpha^{\prime \prime}+\alpha\right) V\left(\frac{i_{0} i_{x} i_{2}}{\Delta}\right)=\alpha \alpha^{\prime} \alpha^{\prime \prime} \Delta$.

Daraus ergiebt sich :

oder

$$
i_{0}+i_{1}+i_{2}+2 V\left(\frac{i_{0} i_{1} i_{2}}{\Delta}\right)=\Delta
$$

oder endlich :

$$
4 i_{\mathrm{o}} i_{\mathrm{s}} i_{\mathrm{g}}=8 \mathrm{~g} \Delta,
$$

Man kann also aus dem Inhalte der drei kleinen Dreiecke den Inhalt des grolsen finden.

12. Halbirt man die Linien $A_{0} J_{0}, A_{1} J_{1}, A_{2} J_{2}$ in den Puncten $B_{0}, B_{1}, B_{z}$, so sind die Coordinaten dieser Puncte: 

$\left(B_{0}\right) \quad \xi^{(0)}=\frac{\alpha^{\prime} \alpha^{\prime} x^{\prime}-\alpha^{\prime \prime} \alpha^{\prime \prime} x^{\prime \prime}}{a^{\prime} \alpha^{\prime}-\alpha^{\prime \prime} \alpha^{\prime \prime}}$,
$v^{(0)}=\frac{\alpha^{\prime} \alpha^{\prime} y^{\prime}-\alpha^{\prime \prime} \alpha^{\prime \prime} y^{\prime \prime}}{\alpha^{\prime} \alpha^{\prime}-\alpha^{\prime \prime} \alpha^{\prime \prime}}$,
$\left(B_{1}\right) \quad \xi^{(1)}=\frac{\alpha^{\prime \prime} \alpha^{\prime \prime} x^{\prime \prime}-\alpha \alpha x}{\alpha^{\prime \prime} \alpha^{\prime \prime}-\alpha \alpha}$
$v^{(1)}=\frac{\alpha^{\prime \prime} \alpha^{\prime \prime} y^{\prime \prime}-\alpha \alpha y}{\alpha^{\prime \prime} \alpha^{\prime \prime}-\alpha \alpha}$
$\left(B_{2}\right) \quad \xi^{(2)}=\frac{\alpha a x-\alpha^{\prime} \alpha^{\prime} x^{\prime}}{\alpha \alpha-\alpha^{\prime} \alpha^{\prime}}$,
$\nu^{(z)}=\frac{\alpha \alpha x-\alpha^{\prime} \alpha^{\prime} y^{\prime \prime}}{\alpha \alpha-\alpha^{\prime} \alpha^{\prime}}$

diese drei Puncte liegen demnach auch in einer Geraden.

13. Die halben Entfernungen zwischen den Puncten $A_{0} J_{0}, A_{1} J_{1}$, $A_{2} J_{2}$ seien $R_{0}, R_{1}, R_{2}$, so hat man für rechtwinklige Coordinaten

$$
\begin{aligned}
& R_{0} R_{0}=\left(\frac{\alpha^{\prime} \alpha^{\prime \prime}}{\alpha^{\prime} \alpha^{\prime}-\alpha^{\prime \prime} \alpha^{\prime \prime}}\right)^{2}\left\{\left(x^{\prime}-x^{\prime \prime}\right)^{2}+\left(y^{\prime}-y^{\prime \prime}\right)^{2}\right\} \\
& R_{1} R_{1}=\left(\frac{\alpha^{\prime \prime} \alpha}{\alpha^{\prime \prime} \alpha^{\prime \prime}-\alpha \alpha}\right)^{2}\left\{\left(x^{\prime \prime}-x\right)^{2}+\left(y^{\prime \prime}-y\right)^{2}\right\} \\
& R_{2} R_{2}=\left(\frac{\alpha \alpha^{\prime}}{\alpha \alpha-\alpha^{\prime} \alpha^{\prime}}\right)^{2}\left\{\left(x-x^{\prime}\right)^{2}+\left(y-y^{\prime}\right)^{2}\right\}
\end{aligned}
$$

14. Beschreibt man nun aus dem Puncte $B_{0}$ mit dem Hallomesser $R_{\mathrm{o}}$ einen Kreis, und sind die Coordinaten irgend eines Puncts $Q$ desselben $X, Y$, so ist:

oder :

$$
\left(\xi^{(0)}-X\right)^{2}+\left(v^{(0)}-Y\right)^{2}=R_{0} R_{0},
$$

$$
\begin{aligned}
& \left\{\alpha^{\prime} \alpha^{\prime}\left(x^{\prime}-X\right)-\alpha^{\prime \prime} \alpha^{\prime \prime}\left(x^{\prime \prime}-X\right)\right\}^{2}+\left\{\alpha^{\prime} \alpha^{\prime}\left(y^{\prime}-Y\right)-\alpha^{\prime \prime} \alpha^{\prime \prime}\left(y^{\prime \prime}-Y\right)\right\}^{2} \\
= & \left\{\alpha^{\prime} \alpha^{\prime \prime}\left(x^{\prime}-X\right)-\alpha^{\prime} \alpha^{\prime \prime}\left(x^{\prime \prime}-X\right)\right\}^{2}+\left\{\alpha^{\prime} \alpha^{\prime \prime}\left(y^{\prime}-Y\right)-\alpha^{\prime} \alpha^{\prime \prime}\left(y^{\prime \prime}-Y\right)\right\}^{2},
\end{aligned}
$$

oder, nach einer kurzen Reduction und Division mit $\alpha^{\prime} \alpha^{\prime}-\alpha^{\prime \prime} \alpha^{\prime \prime}$ :

$$
\alpha^{\prime} \alpha^{\prime}\left\{\left(x^{\prime}-X\right)^{2}+(y-Y)^{2}\right\}=\alpha^{\prime \prime} \alpha^{\prime \prime}\left\{\left(x^{\prime \prime}-X\right)^{2}+(y-Y)^{2}\right\},
$$

oder endlich:

$$
\alpha^{\prime} M_{1} Q=\alpha^{\prime \prime}-M_{2} Q \text {. }
$$

Die Entfernungen eines beliebigen Punctes dieses Kreises von den zwei Spitzen $M_{0}$ und $M_{3}$ des Dreiecks stehen also in einem bestiindigen Verhältnifs. Beschreibt man nun eben so aus den Puncten $B_{1}$ und $B_{2}$ mit den Radien $R_{2}$ und $R_{2}$ zwei andere Kreise, so schneiden die beiden ersten sich in einem Puncte $Q^{\prime}$, so dafs

$$
\alpha^{\prime} M_{1} Q^{\prime}=\alpha^{\prime \prime} M_{2} Q^{\prime}=\alpha M_{0} Q^{\prime} \text {; }
$$

eben so die zwei letzten, dafs sie sich also alle drei in einem Puncte schneiden.

15. Für den Fall, dafs $\alpha=\alpha^{\prime}=\alpha^{\prime \prime}$, liegen die Puncte $J_{0}, J_{1}, J_{\dot{1}}$ auf der Mitte der Seiten, die Puncte $A_{0}, A_{1}, A_{2}$ aber in unendlicher Entfernung. Die drei Kreise verwandeln sich also in diesem Falle in drei auf den Seiten aus den Puncten $J_{0}, J_{1}, J_{2}$ errichtete Perpendikel, welche sich demnach in einem Puncte schneiden.

München, den 12. Mai 1830. 